\title{
A Comparative Study of Inguinal Hernia Repair by houldice Method Vs Other Methods
}

\author{
MALATHY . $D^{1}$, Puviarasan.$G^{2}$
}

\begin{abstract}
Background: Hernia is one of the most common surgical conditions encountered in day to day practice. It is a common problem, more in industrial workers who are doing strenuous work over a long period of time. Any method which reduces the recurrence rate as well as lowers the morbidity and post-operative complication rate, must however be considered superior. The common aim in treatment of hernia is to restore the anatomical integrity of the disrupted tissue, performing a strong repair and to prevent further recurrences.

Material and methods: This review was based on the study of 80 selected cases of uncomplicated inguinal hernias treated in our institution by random sampling. All the patients were investigated pre-operative check-up in out-patient clinic for planned surgery. All patients were admitted in our hospital and surgery done under anesthesia (spinal, general, local). All patients in our study received pre-operative antibiotic. In operative technique, the difference lies in the repair of the posterior wall. In the present study comparison of inguinal hernia repair by Shouldice versus other three methods modified Bassini's repair, pre-peritoneal mesh repair and Lichtenstein tension-free repair had been studied with a regular follow up. The selection of the patients for type of anaesthesia was done on the basis of associated cardiac and respiratory diseases.

Results: Out of 80 patients, Shouldice repair was done in 20 patients and other three method of repair was also done in 20 patients each. In present study, wound haematoma occurred in three patients, which may be attributed to the extensive dissection. Seven patients developed wound infection, treated by antibiotics and dressing. Three patients developed scrotal oedema, which was treated by scrotal support and anti-inflammatory agents. Two patients developed urinary retention, relieved by analgesics, hot water bag and ambulation. None of them required catheterization. In the present study we encountered two cases of recurrences (2.5\%).

Conclusion: In Shouldice repair, double breasting ensures the strong repair. In this method of repair minimal tension on the suture line, so relaxing incision are not necessary. Deep inguinal ring is narrowed in the lateral aspect of the repair. Recurrence rate is remarkably low. But the repair is difficult to perform and extensive dissection required and it takes longer operative time.
\end{abstract}

Keywords: Inguinal hernia, Shouldice repair of hernia, Bassini's repair, Pre-peritoneal mesh repair, Lichtenstein tension-free repair.

\section{Introduction}

Hernia is one of the most common surgical conditions encountered in day to day practice. It is a common problem, more in industrial workers who are doing strenuous work over a long period of time [1]. Innumerable techniques have been described and are in use for the repair of inguinal hernia. Any method which reduces the recurrence rate as well as lowers the morbidity and post-operative complication rate, must however be considered superior. The common aim in treatment of hernia is to restore the anatomical integrity of the disrupted tissue, performing a strong repair and to prevent further recurrences. In this study, the repair of adult inguinal hernia by different techniques is compared with the standard series reported in literature. Shouldice, in 1953, had described the multilayered repair and is most successful of "pure tissue" methods with less recurrence rate [2]. It is very difficult to compare the recurrence rate significantly because of short follow up. It has been found from the study that the results of hernia repair depend upon proper selection of cases, skillful operative technique and proper post-operative management.

\section{Aim and objectives}

- X To compare the advantages of one method over the other method.

- X To compare the technical aspects of various methods of inguinal hernia repair.

- X To compare the post-operative convalescence and the recurrence rates.

\section{Material and methods}

This review was based on the study of 80 selected cases of uncomplicated inguinal hernias treated in our institution by random sampling. All the patients were investigated pre-operative check-up in out-patient clinic for planned surgery. All patients were admitted in our hospital and surgery done under anesthesia (spinal, general, local). All patients in our series received pre-operative antibiotic.In operative technique, the difference lies in the repair of the posterior wall. The different methods were as follows. 


\section{Shoudice repair}

An incision is made in the transversalis fascia from internal ring to the pubic tubercle parallel to the inguinal ligament. And flap is made. Multilayer repair is done. First layer of repair is done by suturing the lower lateral flap with deep surface of upper flap. Repair is begun at medial end with the first suture close to the pubic crest but not involving periosteum.

Laterally, last bite medial to the emerging cord creates new internal ring with the underlying transversalis margin below and full thickness of the upper flap above. Second layer of repair is done with the same running suture is used in the reverse direction to create the second layer. The full thickness of the upper flap of transversalis fascia is sutured to the base of the lower edge of transversalis fascia. Sutures should be placed about 2-4 $\mathrm{mm}$ apart and bites of different depth taken. Third layer strated with passing suture through the inguinal ligament at the medial edge of the new internal ring and then takes a bite of the posterior surface of the aponeurotic tendon of the transverses abdominis and is tied. This suture is continued medially upto the pubic tubercle and returns again laterally to suture the anterior sheath and the lower aspect of the conjoined tendon from the front to inner surface of the lower flap of the external oblique aponeurosis. This line is continued back to the internal ring, further reinforceing ring around the emergent cord and suture is tied to its original tail. The cord is now laid on this four layer buttress and the external oblique aponeurosis closed in front of cord in double layer.

\section{Modified bassini's repair}

In this method, the conjoined tendon is sutured to the margin of inguinal ligament by prolene material. The first suture passes through the periosteum of pubic tubercle. Stiches are taken at different level over the inguinal ligament.

\section{Preperitoneal mesh repair}

Abdomen opened via pfannenstiel, midline or suprainguinal incision. Hernia sac reached through pre-peritoneal space. Internally the dissection starts in the paravesical area and must expose successively cooper's ligament, the obturator area and the external iliac vessels. Sac dealt as described previously. Mesh (prolene) fixed with prolene stitches to transversalis fascia, iliopsoas muscle and obturator area.

\section{Lichtenstein tension-free repair}

In this repair a sheet of polypropylene mesh used to reconstruct the entire floor of the inguinal canal without any attempt to close the defect by suture. The mesh is sutured along its lower edge to the pubic tubercle, lacuner ligament and the inguinal ligament beyond the internal ring with a continuous suture of the prolene. The medial edge of mesh is sutured to the rectus sheath, also with prolene continuous suture. The superior edge is fixed with few interrupted sutures with internal oblique muscle. The lateral edge of mesh is slit and the two tails passed around to embrace the cord at the internal ring, then they are crossed over each other and sutured with each other by prolene. This creates a new internal ring and shutter mechanism. The patients were followed up at regular interval and on follow up, the patients were examined for complication, recurrences. All patients were advised not to do any strenuous work for 3 months.

\section{Results and Discussion}

Inguinal hernia is major surgical problem. The various techniques employed for repair of inguinal hernia accounts for various advantages of one technique over the other techniques. Thus, it is mandatory to study and compare the results of various techniques of hernia repair. In the present study, comparison of inguinal hernia repair by Shouldice versus other three methods modified Bassini's repair [3], pre-peritoneal mesh repair [4] and Lichtenstein tension-free repair [5] had been studied with a regular follow up. Majority of patients were between 40-60 years group. Maximum age of patient was 72 year and minimum age was 18 year. In our study, direct inguinal hernia was more common in older age group while indirect inguinal hernia was more common in younger patients which can be comparable with other study [6]. Bilateral hernias were commonly seen in older age group. In present study, all patients having bilateral hernias were above 40 years. According to literature [7], the hernia is bilateral in 12 percent of cases, compared to $25 \%$ in our study as per

Table - 1. Out of 80 cases, $37.5 \%$ had indirect hernia, $58.75 \%$ had direct hernia and $3.75 \%$ had recurrent hernias. $50 \%$ of our patients had right sided hernias, it is comparable to the incidence reported in the series by H. B. Devlin, et al. [8]. The selection of the patients for type of anesthesia was done on the basis of associated cardiac and respiratory diseases. As mentioned in literature, regional or general anesthesia is preferable unless the patient is aged or his general condition is poor, or is suffering from a severe cough or some underlying disease such as diabetes or nephritis, cardiac diseases where local anesthesia may be safely employed [9]. Out of 80 patients, Shouldice repair was done in 20 patients and other three method of repair also was done in 20 patients each as per 
Table - 2. In present study, wound haematoma occurred in three patients, which may be attributed to the extensive dissection. Seven patients developed wound infection, treated by antibiotics and dressing. Three patients developed scrotal edema, which is treated by scrotal support and anti-inflammatory agents. Two patients developed urinary retention, relieved by analgesics, hot water bag and ambulation as per Table - 3 . None of them required catheterization. In the present study, we encountered two cases of recurrences $(2.5 \%)$ as per

Table - 4. As the follow up was too short, recurrence was not as compared to standard series of A. Paul, et al. [10].

Table - 1: Types of hernia.

\begin{tabular}{|l|l|l|}
\hline Types of hernia & Unilateral & Bilaterlal \\
\hline Indirect & 25 & 5 \\
\hline Direct & 32 & 15 \\
\hline Recurrent & 3 & - \\
\hline Pantaloon & - & - \\
\hline
\end{tabular}

Table - 2: Operative techniques.

\begin{tabular}{|c|c|c|c|}
\hline Operative technique & $\begin{array}{l}\text { Indirect } \\
\text { hernia }\end{array}$ & $\begin{array}{l}\text { Direct } \\
\text { hernia }\end{array}$ & $\begin{array}{l}\text { Recurrent } \\
\text { hernia }\end{array}$ \\
\hline Shouldice repair & 5 & 14 & 1 \\
\hline Bassini’s repair & 13 & 7 & - \\
\hline Pre- peritoneal mesh repair & 6 & 12 & 2 \\
\hline Lichtenstein tension-free repair & 6 & 14 & - \\
\hline
\end{tabular}

Table - 3: Post-operative complications.

\begin{tabular}{|l|c|c|}
\hline Post-operative complications & Total & Percentage in present study \\
\hline Wound hematoma & 3 & 3.75 \\
\hline Wound hematoma & 7 & 8.75 \\
\hline Scrotal edema & 3 & 3.75 \\
\hline Deep vein Thrombosis & - & - \\
\hline Chest complication & 1 & 1.25 \\
\hline Retention of urine & 2 & 2.5 \\
\hline
\end{tabular}

Table - 4: Recurrence.

\begin{tabular}{|l|c|c|c|}
\hline $\begin{array}{l}\text { Type of hernia } \\
\text { repair }\end{array}$ & Total & Recurrence & $\begin{array}{l}\text { Recurrence } \\
\text { (Percentage) }\end{array}$ \\
\hline Shouldice repair & 20 & - & - \\
\hline Bassini's repair & 20 & 1 & 5 \\
\hline $\begin{array}{l}\text { Pre- peritoneal mesh } \\
\text { repair }\end{array}$ & 20 & 1 & 5 \\
\hline $\begin{array}{l}\text { Lichtenstein } \\
\text { tension-free repair }\end{array}$ & 20 & - & - \\
\hline
\end{tabular}

\section{Conclusion}

In Shouldice repair, double breasting ensures the strong repair. In this method of repair minimal tension on the suture line, so relaxing incision are not necessary [11]. Deep inguinal ring is narrowed in the lateral aspect of the repair. Recurrence rate is remarkably low [12]. But the repair is difficult to perform and extensive dissection required and it takes longer operative time [13].

\section{References}

[1]. Ashindoitiang JA, Ibrahim NA, Akinlolu OO. Risk factors for inguinal hernia in adult male Nigerians: a case control study. Int J Surg., 2012; 10: 364-7.

[2]. Amato B, Moja L, Panico S, Persico G, Rispoli C, Rocco N, Moschetti I. Shouldice technique versus other open techniques for inguinal hernia repair. Cochrane Database Syst Rev., 2012; 4: CD001543.

[3]. Economopoulos KJ, Milewski MD, Hanks JB, Hart JM, Diduch DR. Sports hernia treatment: modified bassini versus minimal repair. Sports Health, 2013; 5: 463-9.

[4]. Maw A. Peer review report on Long- term outcome for open preperitoneal mesh repair of recurrent inguinal hernia. Int J Surg., 2015; 30: 13 .

[5]. Rönkä K, et al. Randomized Multicenter Trial Comparing Glue Fixation, Self- gripping Mesh, and Suture Fixation of Mesh in Lichtenstein Hernia Repair (FinnMesh Study). Ann Surg., 2015; 262: 714-20. 
[6]. Vassiliadis A, et al. Inguinal Hernia: Lap vs Open. Hernia, 2015; 19: S57-62.

[7]. Sharma D, Yadav K, Hazrah P, Borgharia S, Lal R, Thomas S. Prospective randomized trial comparing laparoscopic transabdominal preperitoneal (TAPP) and laparoscopic totally extra peritoneal (TEP) approach for bilateral inguinal hernias. Int J Surg., 2015; 22: 110-7.

[8]. Devlin HB, Russell IT, Muller D, Sahay AK, Tiwari PN. Short-stay surgery for inguinal hernia. Clinical outcome of the Shouldice operation. Lancet, 1977; 1: 847-9

[9]. Bakota B, Kopljar M, Baranovic S,Miletic M, Marinovic M, Vidovic D. Should we abandon regional anesthesia in open inguinal hernia repair in adults? Eur J Med Res., 2015; 20: 76.

[10]. Paul A, et al. Randomized trial of modified Bassini versus Shouldice inguinal hernia repair. The Cologne Hernia Study Group. Br J Surg., 1994; 81: 1531-1534.

[11]. N N, R S. A Comparative Study between Modified Bassini's Repair and Lichtenstein Mesh Repair (LMR) of Inguinal Hernias in Rural Population. J Clin Diagn Res., 2014; 8: 88-91.

[12]. Liu Z, et al. Comparison of open preperitoneal repair and Lichtenstein herniorraphy on the surgical treatment of inguinal hernia. Zhonghua Wai Ke Za Zhi, 2014; 52(9): 682-5.

[13]. Chatzimavroudis G, et al. Lichtenstein technique for inguinal hernia repair using polypropylene mesh fixed with sutures vs. self-fixating polypropylene mesh: a prospective randomized comparative study. Hernia, 2014; 18: 193-8 